3. The stability of the nonswitch RT over RSI is important for the logic of using the short/switch and long/nonswitch conditions as the basis distributions. However, the increase in error rate with RSI on nonswitch trials might suggest an RSI effect that compromises this logic. There seem to be three possibilities. (1) This trend reflects a process restricted to error generation (e.g., occasional "lapses" of concentration); the logic of any test applied to RT distributions is unaffected. (2) The mechanism of this RSI effect applies both to nonswitch trials and to prepared switch trials, in which case the logic of the mixture test is unaffected. (3) A long RSI causes a loss of arousal or readiness on the nonswitch trials only (because the participant has nothing to do but "maintain" the previous task set), but there is also a compensating shift of speed-accuracy tradeoff that counteracts the effect of RSI on RT. Since these two effects cancel out to leave at least the mean of the RT distribution where it ought to be, there is little impact on the logic of the mixture test. Moreover, there is a similar (though admittedly less pronounced) RSI effect on the nonswitch error rate in Rogers and Monsell's (1995) data (Figure 1, bottom left panel). Thus, even if this effect reflects something that causes both experiments slightly to misestimate $\alpha$, the important effect of experiment on $\alpha$ should remain relatively unaffected.

(Manuscript received August 15, 2000; revision accepted for publication April 25, 2001.)

\title{
SCiP 2002 \\ Call for Papers
}

The 32nd annual meeting of the Society for Computers in Psychology will be held at the Hyatt hotel (Kansas City, MO) on November 21, 2002, before the opening of the annual meeting of the Psychonomic Society. Typically, the program includes posters, papers, symposia, and tutorials dealing with the use of computers in all areas of psychology, including research, education, and industry. Sessions will provide an opportunity for experienced computer users to exchange information on various aspects of computing and for less experienced users to attend tutorials. In addition, there will be invited addresses of general interest and commercial exhibits. Instructions for submitting papers and examples of sessions at recent conferences can be found at the SCiP website: http://www.lafayette.edu/allanr/scip.html.

Specific topics suggested for this year's conference include, but are in no way limited to, research and teaching on the Web, modeling of cognitive processes, and computer-based tools for research. As usual, articles based on papers presented at the SCiP meeting will be invited for an issue of Behavior Research Methods, Instruments, \& Computers that focuses on the conference.

You need not be a member of SCiP to present at the conference (although you are certainly encouraged to join). Presentations have traditionally been talks of 15 to 20 minutes. Symposia are groups of about 4 to 6 talks on a related topic. If you are interested in organizing a symposium, or have a suggestion for a symposium that you would like to see organized, please contact the program chair as early as possible. If you are interested in presenting a talk or poster, you must submit your materials by the deadline of June 28th, 2002 .

If computers have become integral to your work as a psychologist-or if you'd like to learn more about using technology to improve your instruction, research, communication, and analyses-come to Kansas City and join us for a stimulating day of presentations, demonstrations, and tutorials!

You are encouraged to contact Robert W. Proctor (program chair) with questions or comments.

Address: Department of Psychological Sciences, Purdue University, West Lafayette, IN 47907-1364.

E-mail: proctor@psych.purdue.edu. 